Sympozjum

Rok XXIII 2019, nr 1(36), s. 205-224

\author{
ks. Adam Pastorczyk SCJ \\ Uniwersytet Papieski Jana Pawła II w Krakowie \\ ORCID: 0000-0002-0810-3878; e-mail: scj.adam@gmail.com \\ https://doi.org/10.4467/25443283SYM.19.012.10641
}

\title{
LA SYNAXIS LITURGICA NELLA COSCIENZA DELLA CHIESA PRIMITIVA SULLA BASE DELLA RILETTURA DI IOANNIS ZIZIOULAS
}

\author{
LITURGICZNA SYNAKSA W ŚWIADOMOŚCI \\ PIERWOTNEGO KOŚCIOŁA NA PODSTAWIE RELEKTURY \\ IOANNISA ZIZIOULASA
}

\section{THE LITURGICAL SYNTAX IN THE CONSCIOUSNESS OF EARLY CHURCH BASED ON THE RELECTURE OF IOANNIS ZIZIOULAS}

\begin{abstract}
Abstrakt
Przedmiot naszej pracy stanowi zbadanie pojęcia liturgicznej synaksy w świadomości pierwotnego Kościoła na podstawie relektury Pisma i Tradycji dokonanej przez Ioannisa Zizioulasa. W odniesieniu do Pisma, nasze badanie będzie opierało się przede wszystkim na relekturze Pierwszego Listu św. Pawła do Koryntian. Interpretacja biblijnych treści w optyce naszego autora pozwoli nam na wyróżnienie dwóch podstawowych aspektów starożytnej wspólnoty eucharystycznej (geograficznego i eschatologicznego), kształtujących eklezjologiczno-eucharystyczne pojęcie liturgicznej synaksy pierwszych wieków chrześcijaństwa. W patrystyce, zgodnie ze wskazówkami Zizioulasa, wymiarów liturgicznej synaksy Kościoła pierwotnego poszukiwać będziemy u Ignacego z Antiochii w jego zbudowa-
\end{abstract}


nym na osobie biskupa eucharystycznym modelu Kościoła, u Justyna Męczennika, naocznego świadka eucharystycznej wspólnoty drugiego wieku, a także u Maksyma Wyznawcy, prezentującego ikoniczną perspektywę życia wspólnoty liturgicznej.

Słowa kluczowe: Ioannis Zizioulas, liturgiczna synaksa, teologia prawosławna, Kościół pierwotny

\section{Abstract}

The subject of our work is to examine the concept of liturgical syntax in the consciousness of early Church based on the relecture of the Scripture and Tradition by Ioannis Zizioulas. With regard to the Scriptures, our study will be based primarily on the relecture of the First Letter of Saint Paul to the Corinthians. The interpretation of biblical content in the author's optics will allow us to distinguish two basic aspects of the ancient Eucharistic community (geographical and eschatological), shaping the ecclesiological-eucharistic concept of the liturgical syntax in the first centuries of Christianity. In the patristic, according to Zizioulas'research, the liturgical dimensions of the syntax of early Church will be sought by Ignatius of Antioch in his Episcopal Bishop model of the Church, with Justin Martyr, an eyewitness of the Eucharistic community of the second century, and Maximus the Confessor, presenting the iconic perspective of living liturgical community.

Keywords: Ioannis Zizioulas, liturgical syntax, Orthodox theology, early Church

\section{Le testimonianze del Nuovo Testamento}

La „Chiesa locale” dell'antichità cristiana ha strutturato la sua identità all'interno del contesto eucaristico, che ha determinato fondamentalmente la sua esistenza come corpo di Cristo ${ }^{1}$. Logicamente, l'approccio ecclesiologico dellepoca non era mai espresso attraverso la speculazione in-

${ }^{1}$ Cfr. I. Zizioulas, Being as Communion. Studies in Personhood and the Church, London 1985, 247. 
tellettuale, ad esempio con qualche trattato teoretico, bensì nella prassi, nella synaxis liturgica, la quale non può che essere esaminata sotto due principi basilari: la sua natura geografica e la sua cattolicità․ A tal proposito, il nostro percorso sarà guidato (nella sua fase primaria) dagli scritti paolini (prevalentemente 1 Cor), i quali, almeno in parte, venivano letti durante la celebrazione liturgica ${ }^{3}$. La loro rilettura in chiave zizioulana, ci permetterà di rivedere esplicitamente la nozione ecclesiologico-eucaristica, presente nella coscienza della Chiesa primitiva.

\subsection{La natura geografica ed eucaristica della synaxis liturgica (1 Cor)}

„Paolo, apostolo di Gesù Cristo per volontà di Dio, e il fratello Timoteo, alla Chiesa di Dio che è in Corinto e a tutti i santi dell'intera Acaia" $(2 \text { Cor } 1,1)^{4}$. Già solo un rapido sguardo del saluto paolino fa notare una certa distinzione nell'uso dei vocaboli. Nei riguardi della città viene usata la parola „Chiesa”, invece quando l'apostolo si riferisce agli abitanti di tutta l'area geografica dell'Acaia, li chiama „santi”. Tale fenomeno linguistico non avrebbe attirato la nostra attenzione se non fosse ampiamente presente nelle altre lettere paoline. Se leggiamo ad esempio Rm 16,1; Col 4,16 e 1 Ts 1,1, notiamo subito l'uso del termine „Chiesa” al singolare, come nel caso della già menzionata comunità dei Corinzi. Al contrario, quando riflettiamo su Gal 1,2; 1 Ts 2,14; 2 Cor 8,1 e Rm 16,16 , ci rendiamo conto che la parola „Chiesa” viene applicata al plurale (la situazione dei santi dell'intera Acaia) ${ }^{6}$. Ciò vuol dire che l'apostolo utilizzava consapevolmente sia l'una sia l'altra forma nel modo inteso ${ }^{7}$. Il nostro compito sarà quello di dare ragione di questaffermazione.

2 Cfr. Idem, Lessere ecclesiale, Magnano 2007, 249.

3 Cfr. Idem, Eucharist, Bishop, Church. The Unity of the Church in the Divine Eucharist and the Bishop During the First Three Centuries, Brookline 2001, 46.

4 Tutte le citazioni sono tratte dal testo della La sacra Bibbia della Conferenza Episcopale Italiana, Città del Vaticano 2008.

5 Cfr. I. Zizioulas, Eucharist, Bishop, Church, op. cit., 46-47.

${ }^{6}$ Cfr. ibid., 69, note 2 e 3.

7 Cfr. I. Zizioulas, The Local Church in a Eucharistic Perspective. An Orthodox Contribution, „Mid-stream” 33 (1994), 422. 
Per questo motivo, ci riferiamo di nuovo alla Chiesa di Corinto, alla quale Paolo, nella sua prima lettera, scriveva: „(...) quando vi radunate in assemblea, vi sono divisioni tra voi $(. .$.$) " (1 Cor 11,18)$. La rilettura zi-

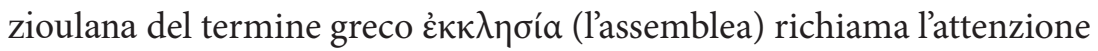
sul reale ed attuale incontro della comunità dei credenti. Così, il nostro teologo esclude qualunque possibilità di interpretare l'adunanza cristiana in chiave psicologica e teoretica. Non lo preoccupano le speculazioni teoretiche dellepoca moderna perché lecclesiologia dell'antichità cristiana si basava più sulle realtà da tutti sperimentabili che sui concetti astratti. Non siamo una Chiesa quando per una qualunque ragione ci incontriamo in qualsiasi tempo ed in qualsiasi luogo? Interrogativi di questo tipo venivano automaticamente respinti ${ }^{8}$. Il pragmatismo ecclesiale spinge Zizioulas ad affermare che la Chiesa non è nient'altro che l'esistenza della comunità concreta, convocata per uno scopo preciso e fisicamente presente nei suoi membri in un unico luogo: „è anzitutto qualcosa che viviamo e vediamo, e non tanto qualcosa che definiamo con parole".

Quale è il motivo per cui i cristiani si radunano in un luogo, più frequentemente in una certa città? ${ }^{10}$.

Paolo stesso nei successivi versetti della Prima Lettera ai Corinzi che abbiamo appena esaminato, affronta nel modo diretto la nostra questione: "Quando dunque vi radunate insieme, il vostro non è più un mangiare la cena del Signore. Ciascuno infatti, quando partecipa alla cena, prende prima il proprio pasto e così uno ha fame, l'altro è ubriaco" (1 Cor 11,20-21). Andando avanti leggiamo: „(...) chi mangia e beve senza riconoscere il corpo del Signore, mangia e beve la propria condanna" (1 Cor 11,29) ${ }^{11}$.

${ }^{8}$ Cfr. Idem, Eucharist, Bishop, Church, op. cit., 48.

9 Y. SPITERIs, Ecclesiologia ortodossa. Temi a confronto tra Oriente e Occidente, Bologna 2003, 68. Il testo viene dalle non pubblicate lezioni di Zizioulas tenute all'Università di Tessalonica nell'anno 1990-1991.

${ }^{10}$ Cfr. I. Zizioulas, Eucharist, Bishop, Church, op. cit., 94. Zizioulas ricorda che inizialmente il cristianesimo era una religione di città. Cfr. IDEM, The Early Christian Community, in: B. McGinn, J. Meyendorff, J. Leclercq (ed.), Christian Spirituality. Origins to the Twelfth Century, New York 1986, 30.

${ }^{11}$ Cfr. I. Zizioulas, The Ecclesiological Presuppositions of the Holy Eucharist, „Nicolaus"10 (1982), 334, nota 1. 
La rivelazione del carattere eucaristico del raduno ecclesiale passa attraverso il racconto paolino sugli abusi dei Corinzi che avrebbero luogo durante la liturgia. L'apostolo stigmatizza in termini molto chiari gli scandalosi e indegni comportamenti dei cristiani; inoltre, approfittando della circostanza, continua la sua riflessione e identifica a tal punto l'Eucaristia con la Chiesa di Dio, per cui l'offesa verso la prima getta il disprezzo anche sull'ultima (1 Cor 11,22).

La stessa coincidenza eucaristico-ecclesiale la troviamo pure nel versetto seguente: „Io, infatti, ho ricevuto dal Signore quello che a mia volta vi ho trasmesso" (1 Cor 11,23), ossia, la celebrazione eucaristica ${ }^{12}$. In tal

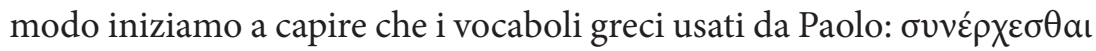

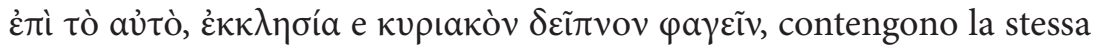
realtà, sia quella eucaristica che quella ecclesiale ${ }^{13}$. Per questa ragione, d'ora in poi useremo questi concetti in maniera intercambiabile: pensando all'Eucaristia non dovremo dimenticare il suo carattere ecclesiale e, viceversa, quando prenderemo in considerazione l'idea della Chiesa ci riferiremo necessariamente all'Eucaristia ${ }^{14}$.

Da quanto precede risulta che la lettera, il cui carattere eucaristico è stato già delineato, poteva essere letta soltanto nel seno di assemblee liturgiche già esistenti. Come abbiamo detto, tali adunanze, nella fase iniziale dell'antichità cristiana, si tenevano esclusivamente nelle città. Quanti abitavano in territori periferici raggiungevano i centri abitati più importanti: ad esempio i cristiani dell'Acaia venivano convocati a Filippi, quelli della Macedonia a Corinto e quelli dell'Anatolia ad Efeso. Per questo motivo si può affermare che al tempo dell'attività apostolica di Paolo non esistesse alcun'opera che provasse la presenza di una Chiesa regionale ${ }^{15}$.

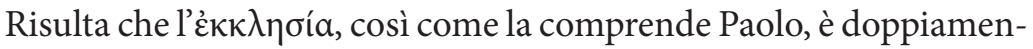
te contestualizzata. In primo luogo dev'essere vista in chiave geografica:

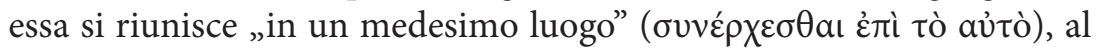
fine di radunare tutte le genti, come se fosse "una rete gettata nel mare,

${ }^{12}$ Cfr. Idem, Eucharist, Bishop, Church, op. cit., 48.

${ }^{13}$ Cfr. Idem, The Ecclesiological Presuppositions of the Holy Eucharist, op. cit., 334.

${ }^{14}$ Cfr. ibid., 343.

${ }^{15}$ Cfr. I. Zizioulas, Eucharist, Bishop, Church, op. cit., 47. 
che raccoglie ogni genere di pesci” (Mt 13,47) ${ }^{16}$. In secondo luogo non può che essere letta in chiave liturgica, strettamente connessa con l'Eu-

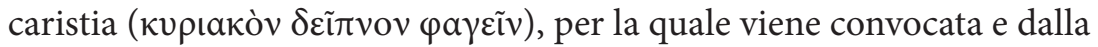
quale riceve la sua propria identità, ossia quella cattolica.

\subsection{La natura escatologica della synaxis liturgica

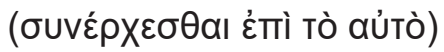

Lo sguardo, che abbiamo appena rivolto alla natura geografica ed eucaristica della synaxis liturgica, permette di cogliere l'importanza che $\mathrm{i}$ cristiani dei primi secoli hanno accordato a questo evento accaduto in un luogo (hic) e in un tempo (nunc) ben precisi ${ }^{17}$. Tuttavia, laffermare che essi si radunavano per la celebrazione eucaristica esprime soltanto un aspetto di questa realtà. Infatti, la loro mentalità, influenzata biblicamente, aveva come suo fondamento l'avvenimento escatologico ${ }^{18}$.

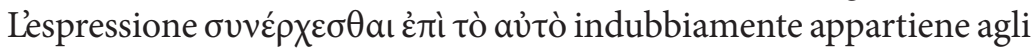
embrionali tentativi di riflessione sulla Chiesa locale a sfondo escatologico $^{19}$. La coincidenza della dimensione spaziale con l'eschaton e con la parusia, secondo Zizioulas, è visibile già a partire dagli scritti veterotestamentari, soprattutto dal libro del profeta Daniele. La sua visione esprime le speranze anticotestamentarie che il popolo non disperso, ma radunato Ėđì tò aủtò, ripone nell'apparizione del Figlio dell'uomo sulle nubi del cielo ${ }^{20}$ : „[il vegliardo] gli diede potere, gloria e regno; tutti i popoli, nazioni e lingue lo servivano; il suo potere è un potere eterno, che non tramonta mai, e il suo regno è tale che non sarà mai distrutto" (Dn 7,14).

Zizioulas trasferisce il concetto giudaico all'ambiente cristiano con l'aiuto di Rudolf Schnackenburg, secondo il quale anche la Chiesa primitiva nutriva simili aspettative escatologiche: „non è sostenibile nella co-

${ }_{16}$ Cfr. Idem, Eucaristia e regno di Dio, Magnano 1996, 23.

17 Cfr. Idem, The Early Christian Community, op. cit., 23.

18 Cfr. ibid., 29.

19 Cfr. E. Brancozzi, Chiesa locale, „Firmana” 2 (2010), 28; A. Papanikolaou, Being with God. Trinity, Apophaticism and Divine-Human Communion, Notre Dame (IN) 2006, 34. ${ }^{20}$ Cfr. Y. SpITERIS, Ecclesiologia ortodossa, op. cit., 70. 
munità post-pasquale una cristologia senza attesa della parusia"21. Quest'attestazione necessariamente richiama l'assemblea dell'ultimo giorno, radunata „nello stesso luogo”, dove „saranno riunite davanti a lui tutte le genti" (Mt 25,32a).

Sulla base degli scritti neotestamentari, Zizioulas riconosce un forte

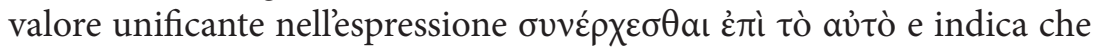
„non è causale il fatto che assai presto l'Eucaristia come icona del regno venga descritta come «sinassi», riunione «in un medesimo luogo»"22.

È la morte di Cristo a possedere carattere unificante; questo è il pensiero di Zizioulas. Nell'evangelo di Giovanni, il sommo sacerdote Caifa profetizza che la finalità della passione di Gesù, non porta con sé semplicemente un valore salvifico ma anche il potere di unire ${ }^{23}$ : „Gesù doveva morire per la nazione e non per la nazione soltanto, ma anche per riunire insieme i figli di Dio che erano dispersi" (Gv 11,52). Inoltre, nel sesto capitolo dello stesso vangelo, l'adunanza dell'ultimo giorno viene espressa con l'aiuto della narrazione sulla moltiplicazione dei pani, che si conclude con la proclamazione del Cristo „veniente” ${ }^{24}$ : „(..) raccolsero [i pezzi avanzati] e riempirono dodici canestri (...)” (Gv 6,13).

Concludiamo le citazioni del Nuovo Testamento con un testo della Chiesa primitiva, l'Insegnamento degli Apostoli. Come ci ricorda Zizioulas, esso contiene la più antica preghiera eucaristica, la quale ci offre una giusta ed esplicita applicazione dei testi neotestamentari alla Chiesa dispersa e radunata: „Come questo pane spezzato era sparso sui colli e raccolto divenne una cosa sola, così la tua Chiesa si raccolga dai confini della terra nel tuo regno, poiché tua è la gloria e la potenza per Gesù Cristo nei secoli”25.

21 R. Schnackenburg, Cristologia del Nuovo Testamento, in: F. V. Joannes (ed.), Mysterium Salutis. Nuovo corso di dogmatica come teologia della storia della salvezza, III/I, Brescia 1971, 325. Si può vedere pure lo stesso Schnackenburg in Die Sittliche Botschaft des Neuen Testaments. Von Jesus zur Kirche, I, Freiburg 1986, 176-187.

${ }^{22}$ I. Zizioulas, Eucaristia e regno di Dio, op. cit., 23-24.

23 Cfr. ibid., 23.

24 Cfr. ibid., 24.

${ }^{25}$ Didachè, in: A. Quacquarelli (ed.), I Padri apostolici, Roma $1998^{9}$ (Testi Patristici 5), IX,4. 


\section{La sintesi patristica greca}

L'esperienza ecclesiale presente nel dato biblico viene adesso posta in evidenza dai Padri. I loro scritti, secondo Zizioulas, si presentano come un mosaico di immagini e concetti e, nonostante provengano da diversi contesti culturali e politici in cui erano stati elaborati, dimostrano una specie di continuità ed integrità del pensare la Chiesa in chiave biblica ${ }^{26}$.

\subsection{Ignazio di Antiochia}

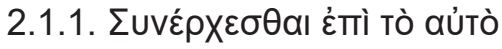

I dati biblici non lasciano dubbi circa il fatto che la Chiesa radunata ,in un medesimo luogo" possiede una dimensione escatologica. Secondo Zizioulas, nelle Apologie di Giustino, questa si sarebbe conservata nella coscienza ecclesiale e di conseguenza nella sua prassi liturgica, almeno fino alla metà del secondo secolo ${ }^{27}$.

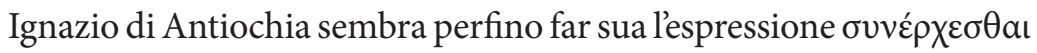

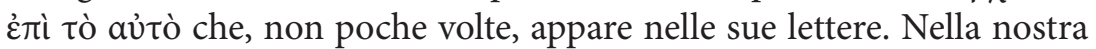
presentazione, con Zizioulas, la esaminiamo in due delle lettere di Ignazio di Antiochia: la Lettera agli Efesini e la Lettera ai Magnesii.

Alla fine della prima lettera, Ignazio, volendo sottolineare ciò che per lui è più importante e ciò che dovrebbe richiedere una particolare attenzione da parte degli Efesini, scrive: „(...) ognuno e tutti insieme nella grazia che viene dal suo nome vi riunite in una sola fede (...) in una concordia stabile spezzando l'unico pane che è rimedio di immortalità (...)" (Lettera agli Efesini XX,2) ${ }^{28}$. Alcuni teologi hanno interpretato questo testo come se Ignazio avesse introdotto nella vita eccle-

${ }^{26}$ Cfr. F. Clancy, Trinity and Ecclesiology. Need for a Patristic Ressourcement, in: D. Marmion, G. Thiessen (ed.), Trinity and Salvation. Theological, Spiritual and Aesthetic Perspectives, Bern 2009, 61.

27 Cfr. I. Zizioulas, Eucharist, Bishop, Church, op. cit., 93.

${ }_{28}$ Citiamo questa e le seguenti lettere di Ignazio di Antiochia dall'edizione critica: A. Quacquarelli (ed.), I Padri apostolici, Roma $1998^{9}$. 
siale una certa forma di paganesimo (un sacramentalismo tipicamente magico). In questo senso, l'Eucaristia conterrebbe il potere soprannaturale dell'immortalità. In realtà, come spiega Zizioulas, è l'esatto contrario, perché l'Eucaristia, nella teologia ignaziana, significa soprattutto

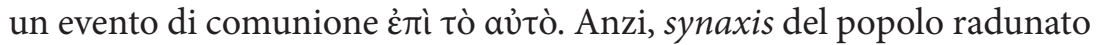
nell'attesa della parusia. In questo senso, „rimedio di immortalità” è la partecipazione stessa all'unica synaxis liturgica della Chiesa ${ }^{29}$, che si tiene simultaneamente con quella celeste.

L'unione ecclesiale è così importante per Ignazio che egli non esita a dire che chi si oppone ad essa e „chi non partecipa alla riunione è un orgoglioso e si è giudicato" (Lettera agli Efesini V,3). Ne consegue che il pensiero dell'Antiocheno, secondo Zizioulas, è pienamente conforme al concetto biblico di salvezza, la quale non è pensabile individualmente, bensì soltanto in maniera ecclesiale $e^{30}$. La concezione ignaziana della synaxis liturgica, condivisa fortemente da Zizioulas, viene anche sostenuta da J. A. Möhler, un altro grande teologo della Chiesa, per il quale „la vita del cristiano è una cosa sola con la vita della Chiesa, perché da quella fluisce, né può essere separata" ${ }^{31}$.

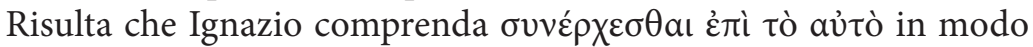
paolino, legandolo strettamente alla prassi liturgica. „Un medesimo luogo" reca in sé l'invito all'unico tempio di Dio, intorno all'unico altare (cfr. Lettera ai Magnesii VII,2), e poi, ad una sola preghiera e ad una sola supplica, ad una sola speranza e ad una sola carità; finalmente pure ad una sola mente immersa nella gioia che è Gesù Cristo (cfr. Lettera ai Mag-

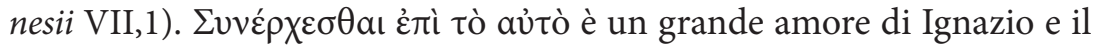
suo desiderio consiste esclusivamente nell'attirarci ad esso: „Impegnatevi a riunirvi più di frequente nell'azione di grazie e di gloria verso Dio" (Lettera agli Efesini XIII,1).

${ }^{29}$ Cfr. I. Zizioulas, The Early Christian Community, op. cit., 31-32.

30 Cfr. ibid., 32.

31 J. A. MöHLER, L'unità nella Chiesa. Il principio del cattolicesimo nello spirito dei $\mathrm{Pa}$ dri della Chiesa dei primi tre secoli, Roma 1969, 30. 


\subsubsection{La cattolicità della Chiesa locale fondata sulla figura} del vescovo indispensabile alla synaxis liturgica

Una Chiesa locale non può essere chiamata Chiesa cattolica ( $\kappa \alpha \theta \theta_{\iota} \iota \kappa \eta$ $\dot{\varepsilon} \kappa \kappa \lambda \eta \sigma i ́()$ solo sulla base dell'essere radunata „in un medesimo luogo”, ma essa deve pure esprimere pienamente ciò che compie mentre celebra l'Eucaristia. In altri termini, il raduno eucaristico deve necessariamente soddisfare la condizione del superamento di tutte le divisioni, includendo in sé tutti i membri di un certo luogo: „La coscienza del carattere cattolico dell'assemblea eucaristica risulta dalla sua unicità in una città e dal suo carattere inclusivo delle diverse categorie di persone appartenenti alla comunità"32.

Se vogliamo esaminare il concetto della cattolicità della Chiesa locale, nella coscienza della Chiesa primitiva, dobbiamo fare due passi: in primo luogo, è necessario considerare brevemente la cattolicità nel suo contesto naturale, quello greco (aristotelico), dal quale viene trasferito agli scritti cristiani; in secondo luogo, dovremo analizzare le lettere di Ignazio di Antiochia indirizzate alle diverse Chiese locali, dove il concetto della cattolicità ecclesiale appare per la prima volta ${ }^{33}$.

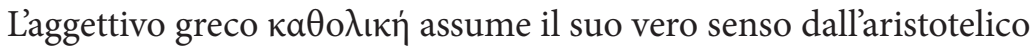

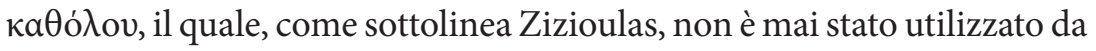
Aristotele per descrivere qualche fenomeno quantitativo (una somma di elementi) o geografico (universale, in tutto il mondo), bensì per esprimere un senso qualitativo (intero, integro, comune). Di conseguenza, non cè bisogno della presenza fisica hic et nunc di tutte le persone per attualiz-

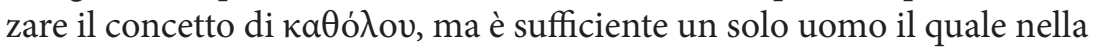
sua forma concreta e personale esprima in un certo luogo ed in un certo tempo tutte le generiche caratteristiche dellumanità ${ }^{34}$.

32 A. MAffeis, La Chiesa locale e l'eucaristia. Lecclesiologia eucaristica nella teologia ortodossa contemporanea, in: G. Canobbio, F. Dalla Vecchia, G. Montini (ed.), La parrocchia come Chiesa locale, Brescia 1993, 167.

${ }_{33}$ Cfr. I. Zizioulas, Eucharist, Bishop, Church, op. cit., 109-110.

${ }^{34}$ Cfr. Aristoteles, De interpretatione, in: F. Didot (ed.), Opera omnia. Graece et latine cum indice nominum et rorum absolutissimo, I, Parisiis 1927, 7,17. Zizioulas si riferisce ad Aristotele in Eucharist, Bishop, Church, op. cit., 109. 


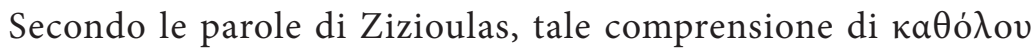
è sopravvissuta fino al tempo della Chiesa. Come essa sia stata accolta ed elaborata dai cristiani dei primi secoli, lo vedremo soprattutto grazie alla Lettera agli Smirnesi: „Come Gesù Cristo segue il Padre, seguite tutti il vescovo e i presbiteri come gli apostoli; venerate i diaconi come la legge di Dio. Nessuno senza il vescovo faccia qualche cosa che concerne la Chiesa. Sia ritenuta valida l'Eucaristia che si fa dal vescovo o da chi è da lui delegato. Dove compare il vescovo, là sia la comunità, come là dove cè Gesù Cristo ivi è la Chiesa cattolica. Senza il vescovo non è lecito né battezzare né fare l'agape; quello che egli approva è gradito a Dio, perché tutto ciò che si fa sia legittimo e sicuro" (Lettera agli Smirnesi VIII,1-2).

La nostra prima osservazione è che il termine "Chiesa cattolica” viene strettamente legato ad un altro concetto, quello di „vescovo", che pure appartiene ai vocaboli basilari del linguaggio ignaziano. Dato che entrambe le parole rappresentano una novità nellambiente ecclesiale dei primi secoli, si è incontrata qualche difficoltà ad interpretarle correttamente. Come ricorda Zizioulas, l'inconsistenza dell'opposizione fra locale e cattolico, così come della nascita del concetto „cattolico” della Chiesa quale risposta all'apparire delle eresie e degli scismi, è un'opinione largamente condivisa tra gli studiosi e che non corrisponde per nulla alla mentalità della Chiesa primitiva ${ }^{35}$. Ciò lo rivela chiaramente il testo sopraccitato (cfr. Lettera agli Smirnesi VIII,1-2), nel quale Ignazio introduce il concetto di cattolicità della Chiesa, riferendosi esclusivamente all'assemblea ecclesiale, senza menzionare minimamente fattori ester$\mathrm{ni}$ - eresie, scismi ${ }^{36}$.

Lintero testo riguarda la persona del vescovo, come punto di partenza e di arrivo: „nessuno senza il vescovo faccia qualche cosa che concerne la Chiesa" (Lettera agli Smirnesi VIII,1). Ė lui che riveste un'importanza cruciale per la cattolicità della Chiesa locale, perché è la figura di Cristo: „Dove compare il vescovo, là sia la comunità, come là dove cè Gesù Cristo ivi è la Chiesa cattolica" (Lettera agli Smirnesi VIII,2). Tale valore

\footnotetext{
${ }^{35}$ Cfr. I. Zizioulas, Lessere ecclesiale, op. cit., 152, nota 4.

${ }^{36}$ Cfr. Idem, Eucharist, Bishop, Church, op. cit., 111.
} 
non gli viene dato a motivo dell'essere semplicemente ordinato come un

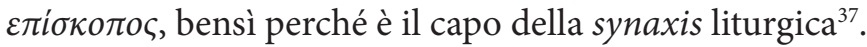

Risulta che la figura del vescovo, che celebra i santi misteri dell'Eucaristia, incarni in sé tutta l'assemblea liturgica. Egli è l'unico e il solo in grado di riunirla in sé per poterla restituire a Cristo, colui che l'ha creata, ed è capace di liberarla dal potere di Satana ${ }^{38}$ : „Quando vi riunite spesso, le forze di Satana vengono abbattute e il suo flagello si dissolve nella concordia della fede" (Lettera agli Efesini XIII,1). In questo modo arriviamo ad un famoso concetto ignaziano: „l' «uno» nei «molti» ed i «molti» nell' «uno»" 39 .

Come spiegare quest'enigmatica espressione e come comprenderla all'interno dell'ecclesiologia ignaziana? I testi, che richiameremo in seguito, non permettono di nutrire alcun dubbio che l'espressione „l' «uno» nei «molti» ed i «molti» nell' «uno»" appartiene al cuore della discussione sulla cattolicità della Chiesa dei primi secoli. Con l'aiuto della Lettera ai Magnesii entriamo nel nostro tema: „Conviene che voi non abusiate dell'età del vescovo, ma per la potenza di Dio Padre gli tributiate ogni riverenza. In realtà ho saputo che i vostri santi presbiteri non hanno abusato della giovinezza evidente in lui, ma saggi in Dio sono sottomessi a lui, non a lui, ma al Padre di Gesù Cristo che è il vescovo di tutti. Per il rispetto di chi ci ha voluto bisogna obbedire senza ipocrisia alcuna, poiché non si inganna il vescovo visibile, bensì si mentisce a quello invisibile. Non si parla della carne, ma di Dio che conosce le cose invisibili” (Lettera ai Magnesii III,1-2).

Il testo ignaziano a prima vista può sembrare scioccante a causa della stretta identificazione della persona del vescovo con Dio stesso. Ignazio non esita a dire che tutto ciò che riguarda il vescovo visibile, riguarda pure il vescovo invisibile. Il vescovo, mentre svolge la funzione di capo della synaxis, è una figura di Cristo e del Padre; un'immagine non soltanto in senso figurato e simbolico, ma in senso reale ${ }^{40}$.

\footnotetext{
37 Cfr. IDEM, The Early Christian Community, op. cit., 32.

${ }^{38}$ Cfr. IDEM, L'essere ecclesiale, op. cit., 164.

39 Cfr. IDEm, Eucharist, Bishop, Church, op. cit., 115.

40 Cfr. ibid., 116.
} 
In tale maniera, spiega Zizioulas, nellecclesiologia ignaziana viene introdotto il concetto di icona, che, nel quarto secolo, sarà espresso da Basilio il Grande nella seguente formulazione: „Allo stesso modo che lautorità e il potere che ci regge è uno solo, così anche unica è la gloria che eleviamo, e non molte, poiché l'onore reso all'immagine trapassa al prototipo" ${ }^{\prime 1}$.

Lecclesiologia ignaziana è quindi profondamente tipologica ${ }^{42}$. Il vescovo, come alter Christus, unisce in sé sia la comunità locale e sia quella cattolica. Ciò è possibile perché nella santa Eucaristia e nella persona del vescovo, intimamente legata alla celebrazione eucaristica, la Chiesa particolare contiene tutta la presenza di Cristo. In questo senso,

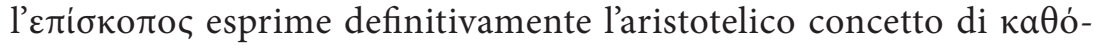
$\lambda$ ov, applicandolo alla relazione fra locale e cattolico ${ }^{43}$ : come per Aristotele ogni uomo rappresenta una incarnazione viva di tutta l'umanità, così per Ignazio la Chiesa locale attualizza hic et nunc l'esistenza della Chiesa cattolica ${ }^{44}$.

In conclusione, insieme alla consapevolezza dell'unicità, ,uno il calice nell'unità del suo sangue, uno è l'altare come uno solo è il vescovo" (Lettera ai Filadelfiesi IV), cresceva nella comunità eucaristica anche la coscienza della totalità, la quale appartiene alleterna volontà del Padre, „(...) il disegno cioè di ricapitolare in Cristo tutte le cose, quelle del cielo come quelle della terra" (Ef 1,10). Ignazio, seguendo i passi di Paolo, ne è un testimone credibile. Come sottolinea Zizioulas, la visione ignaziana di Chiesa costituiva la base per gli altri documenti ecclesiali dell'epoca. Conseguentemente, lecclesiologia e la spiritualità cristiana dei primi secoli erano sotto la sua ispirazione ${ }^{45}$.

\footnotetext{
41 Basilio di Cesarea, Lo Spirito Santo, Roma 1993 (Testi Patristici 106), XVIII, 45.

42 Cfr. ibid., 72.

${ }^{43}$ Cfr. I. Zizioulas, Eucharist, Bishop, Church, op. cit., 117.

${ }^{44}$ Cfr. ibid., 118.

${ }^{45}$ Cfr. I. Zizioulas, The Early Christian Community, op. cit., 32.
} 


\subsection{Giustino}

A quanto pare, non soltanto il radunarsi insieme, in un medesimo luogo" a causa dell'Eucaristia e sotto la presidenza del vescovo, ma pure il farlo nel tempo pasquale, aveva la sua importanza ed evocava l'essenziale dimensione escatologica della Chiesa. „La più eloquente dimostrazione del carattere escatologico dell'Eucaristia [della Chiesa] e della sua identificazione con la pregustazione del regno di Dio è il fatto che, fin dall'inizio essa fu unita alla domenica quale giorno più idoneo per la sua celebrazione" ${ }^{\text {"46 }}$.

Zizioulas richiama una serie di citazioni su questo argomento ${ }^{47}$, rivolgendosi alla fine a Giustino. Le sue parole sono meritevoli pure della nostra attenzione perché, come quelle paoline, contengono in maniera accurata le più antiche descrizioni della liturgia eucaristica, che viene fondata nel contesto domenicale: „E nel giorno detto del sole, riunendoci tutti in un solo luogo dalla città e dalla campagna, si fa unassemblea e si leggono le memorie degli apostoli e gli scritti dei profeti fino a quando vi è tempo" ${ }^{48}$. Poi „viene portato pane, vino ed acqua e il presidente (...) innalza preghiere e ringraziamenti (...). Dei cibi su cui si è pronunciato il ringraziamento segue la divisione e la distribuzione a ciascuno e per mezzo dei diaconi si mandano a coloro che non sono presenti" ${ }^{49}$.

Ne consegue che, fin dall'inizio della cristianità la domenica manifestava il suo stretto rapporto con la celebrazione eucaristica. È possibile in qualche modo spiegare tale fenomeno ecclesiale? Cosa ha condotto la Chiesa a questa scelta e qual è la base teologica di questo legame?50. Zizioulas legge la testimonianza di Giustino alla luce della risurrezione, cioè della nuova creazione.

${ }^{46}$ IDEM, Eucaristia e regno di Dio, op. cit., 38.

${ }^{47}$ Cfr. ibid., 39.

48 Giustino Martire, Prima Apologia, in: C. Burini (ed.), Gli apologeti greci, Roma 1986, 83-150 (Testi Patristici 59), 67,3.

49 Ibid., 67,5.

${ }^{50}$ Cfr. I. Zizioulas, Eucaristia e regno di Dio, op. cit., 39. 
La domenica, „il primo giorno dopo il sabato” (cfr. Lc 24,1), dunque ,il giorno ottavo" (cfr. Gv 20,26), in realtà è „l'ultimo giorno"51, quello escatologico. La ragione è la seguente: il Risorto apparve di domenica come il primogenito di tutta la creazione (cfr. Col 1,15), per rigenerarla e per farne nuove tutte le cose create ${ }^{52}$. Per Zizioulas, si tratta di una comunità „rivoluzionaria” in un doppio senso: in primo luogo, perché d'ora in poi essa pensa totalmente secondo le categorie del regno e ne esprime i segni: ,amore, gioia, pace, pazienza, benevolenza, bontà, fedeltà, mitezza, dominio di sé" (Gal 5,22); in secondo luogo, perché vive una vita nuova che né cambia né passa. I cristiani non muoiono più, la morte non ha più potere su di loro perché sono „vivi tornati dai morti” (cfr. Rm 6,9). La loro esistenza è immersa pienamente nei novissimi, che, però, non sono separati dalla vita presente, anzi, ne sono il „tutto": „Questo forte movimento verso il futuro, e non semplicemente verso le realtà del cielo, introduce la dimensione temporale dellescatologia" ${ }^{33}$.

\subsection{Massimo il Confessore}

Il percorso patristico concludiamo con l'opera di Massimo il Confessore, nella quale l'ontologia trinitaria viene presentata come una realtà metastorica che, mentre celebra l'Eucaristia, entra nel tempo e comincia ad esserne parte costitutiva. In che modo? Con l'aiuto della comunità stessa, perché d’ora in poi la virtù escatologica viene portata nel mondo nella carne dei cristiani. La verità non può essere chiusa in nessuna rappresentazione mentale ed in nessun individuo, perché essa è sempre più grande delle umane possibilità di ragionare e di comprendere. L'unico modo di procedere è la vita comunionale dei fedeli, che diventa uno specchio della vita intratrinitaria. In questo modo, secondo Zizioulas, Massimo il Confessore intraprende la via della rivelazione scritturistica e della sintesi patristica greca, cioè, dell'identificazione della verità

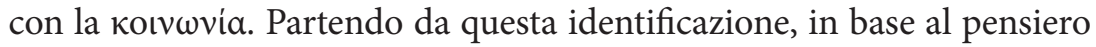

\footnotetext{
51 Cfr. Y. Spiteris, Ecclesiologia ortodossa, op. cit., 70.

52 Cfr. I. Zizioulas, Eucaristia e regno di Dio, op. cit., 40.

53 Ibid., 42.
} 
di Massimo il Confessore, possiamo anche arrivare all'identificazione della verità con l'escatologia: „[L'Areopagita] ha chiamato immagine delle cose vere quanto ora si celebra nella sinassi. (...). Si tratta di simboli, non di verità (...). Dagli effetti: cioè il passaggio da quanto si celebra visibilmente alle realtà invisibili e mistiche, che sono cause e archetipi delle realtà sensibili. Cause, poi, vengono dette quelle realtà che non hanno in alcun modo da altrove le proprietà di essere causa. Ovvero: il passaggio dagli effetti alle cause, cioè dai simboli sensibili alle realtà intelligibili e spirituali, dunque dalle cose più imperfette alle più perfette. Dalle figure, cioè, all'immagine, e da questa alla verità. Ombra [oкı́d] infatti

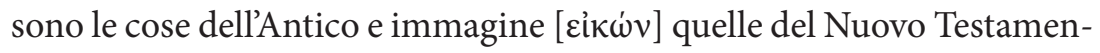
to. Verità $\left[\dot{\alpha} \lambda \hat{n} \theta \varepsilon\llcorner\alpha]\right.$ è la condizione delle cose future" ${ }^{n 4}$.

Il passo di Massimo merita pure particolare attenzione perché mostra l'inversione del concetto di causalità ${ }^{55}$. In concreto, esso si oppone al pensiero dominante nella Grecia antica: „Ombra infatti sono le cose dell'Antico e immagine quelle del Nuovo Testamento". Mentre i platonici hanno dato la priorità logica e temporale alla causa situata nel passato, per il Confessore essa rappresenta soltanto l"'ombra" della verità. Si può dire che lordine delle realtà vere non è convergente con la loro apparenza storica. In breve, più torniamo indietro nel tempo, tanto più entriamo nell'oscurità. La ragione, e dunque la verità stessa, per la quale viene convocata la synaxis, è da trovare unicamente nel futuro ${ }^{56}$.

Notiamo che l'importanza del pensiero del Confessore consiste nell'inversione del tempo. Di conseguenza, per poter riflettere l'archetipo eterno, non dovremmo ricorrere alla fuga dalla storia. Al contrario, la synaxis eucaristica, essendo immersa nel tempo e rimanendo terrena, rappresenta la verità stessa celebrata escatologicamente. Questo non avviene in base alla dispersione e alla fuga, bensì al raduno nell'attesa dell'evento situato nel futuro ${ }^{57}$.

${ }^{54}$ Ibid., 19. Citiamo qui il testo di Massimo il Confessore così come lo presenta Zizioulas.

55 Cfr. I. Zizioulas, Eucaristia e regno di Dio, op. cit., 18.

56 Cfr. ibid., 21.

57 Cfr. ibid., 20. 


\section{I dati emergenti in sintesi}

Dopo aver esaminato le fonti scritturistico-patristiche, nell'ultimo passo ne facciamo una sintesi.

\section{1. $\operatorname{Luogo}$}

Uno dei primi aspetti sui quali Zizioulas getta luce è il concetto di luogo che abbiamo trovato sia nella Bibbia che nella patristica greca. Inoltre, le fonti che abbiamo avuto a nostra disposizione, ci hanno presen-

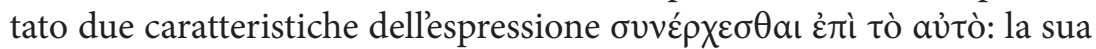
dimensione geografica e quellescatologica. Abbiamo visto che il luogo, uno e concreto, sia per i Giudei che per i cristiani, è parte indispensabile del raduno escatologico.

Zizioulas prende in considerazione questo dato, però, la sua successiva elaborazione andrà piuttosto verso la relazione Spirito Santo-Cristo, cioè, fra il primo (lo Spirito Santo) che convoca i credenti „in un medesimo luogo" e il secondo - Gesù stesso - che diventa luogo par excellence.

\subsection{Vescovo}

Nelle lettere alle sette Chiese dell'Asia, la figura del vescovo viene sì presentata come quella che riunisce in sé la molteplicità dei credenti, però, sempre a causa di Dio, il quale lo fa per primo. Zizioulas entra in questa logica, sviluppando il famoso concetto: „' «uno» nei «molti» ed i «molti» nell' «uno»", riferendolo pienamente a Cristo plasmato dallo Spirito Santo.

Sulla persona del vescovo trova ancora fondamento l'idea dell'essere sacerdote di tutta la creazione. Questo concetto lo abbiamo incontrato nella Lettera agli Efesini XIII,1, però, senza rendercene conto profondamente. Ignazio indicava che un $\varepsilon \pi i \sigma \kappa o \pi о \varsigma$ porta tutta la comunità eucaristica, la sua quotidianità collegata con tutto il mondo, a colui che è capace di liberarla dalle insidie del diavolo.

Zizioulas svilupperà quest' idea, nonostante egli non parli dellessenziale ruolo del vescovo. Si concentrerà piuttosto sul sacerdozio ministeriale in generale e sul sacerdozio di tutti i battezzati. Infatti, la creazione, passando attraverso le mani dell'uomo, sia nella celebrazione eucaristica che nella vita quotidiana dei credenti, vuole essere restituita al suo Creatore. 


\subsection{Tempo}

L"'essere ecclesiale" significa il vivere la domenica. Questo unico giorno è diventato per i cristiani non soltanto il giorno più idoneo per la celebrazione eucaristica, ma pure per la "celebrazione” della loro vita. I cristiani gustano in anticipo il regno e vivono secondo le sue categorie. In questo modo, l'escatologia e la storia coincidono: la prima illumina l'ultima.

La testimonianza patristica indicava ancora il fatto che la synaxis domenicale era rivolta a oriente, come insegnavano le parole dell'epiclesi. Zizioulas prende in considerazione questo dato provato storicamente da Basilio e lo svilupperà successivamente come l'"essere epicletico" della Chiesa.

\subsection{Simbolo ed icona}

L'ultimo dato che proviene dalle testimonianze scritturistico-patristiche e che viene poi elaborato teologicamente da Zizioulas è il concetto iconico e simbolico della synaxis liturgica. In questo caso, appaiono dense di significato le parole zizioulane: „I ministeri che manifestano la comunità escatologica sono talmente fondamentali, da non poter e dover alterarsi, perché se succederà questo, si altererà anche l'essere della Chiesa che si identifica con la comunità escatologica"s8. L'espressione è seria e non meno coraggiosa, perché suppone una stretta identificazione della realtà terrestre con quella escatologica la quale implica l'accettazione di tutte le conseguenze che risultano da questo legame. Sebbene, come dirà Zizioulas, non tutti i ministeri coincidano con la struttura escatologica della Chiesa, le sue parole invitano alla riflessione. Esse ci stimolano a indagare su quali siano gli elementi costitutivi della Chiesa ai quali pensa Zizioulas e, inoltre, sulle conseguenze che una qualsiasi incoerenza può generare fra una comunità e l'altra.

58 Zizioulas in Y. SpITERIs, Ecclesiologia ortodossa, op. cit., 97. 


\section{Bibliografia}

Aristoteles, De interpretatione, in: F. Didot (ed.), Opera omnia. Graece et latine cum indice nominum et rorum absolutissimo, I, Parisiis 1927, 24-38.

Basilio di Cesarea, Lo Spirito Santo, Roma 1993 (Testi Patristici 106).

Brancozzi E., Chiesa locale, „Firmana” 2 (2010), 27-47.

Clancy F., Trinity and Ecclesiology. Need for a Patristic Ressourcement, in: D. Marmion, G. Thiessen (ed.), Trinity and Salvation. Theological, Spiritual and Aesthetic Perspectives, Bern 2009, 11-64.

Didachè, in: A. Quacquarelli (ed.), I Padri apostolici, Roma 1998', 23-39 (Testi Patristici 5).

Giustino Martire, Prima Apologia, in: C. Burini (ed.), Gli apologeti greci, Roma 1986, 83-150 (Testi Patristici 59).

Ignazio di Antiochia, Lettera agli Efesini, in: A. Quacquarelli (ed.), I Padri apostolici, Roma 199899, 99-107 (Testi Patristici 5).

Ignazio di Antiochia, Lettera agli Smirnesi, in: A. Quacquarelli (ed.), I Padri apostolici, Roma 1998', 133-138 (Testi Patristici 5).

Ignazio di Antiochia, Lettera ai Filadelfiesi, in: A. Quacquarelli (ed.), I Padri apostolici, Roma 19989 , 127-132 (Testi Patristici 5).

Ignazio di Antiochia, Lettera ai Magnesii, in: A. Quacquarelli (ed.), I Padri apostolici, Roma 19989 , 109-114 (Testi Patristici 5).

La sacra Bibbia della Conferenza Episcopale Italiana, Città del Vaticano 2008.

Maffeis A., La Chiesa locale e l'eucaristia. L'ecclesiologia eucaristica nella teologia ortodossa contemporanea, in: G. Canobbio, F. Dalla Vecchia, G. Montini (ed.), La parrocchia come Chiesa locale, Brescia 1993, 149-180.

Möhler J. A., L'unità nella Chiesa. Il principio del cattolicesimo nello spirito dei Padri della Chiesa dei primi tre secoli, Roma 1969.

Papanikolaou A., Being with God. Trinity, Apophaticism and Divine-Human Communion, Notre Dame (IN) 2006.

Schnackenburg R. Cristologia del Nuovo Testamento, in: F. V. Joannes (ed.), Mysterium Salutis. Nuovo corso di dogmatica come teologia della storia della salvezza, III/I, Brescia 1971, 289-491. 
Schnackenburg R., Die Sittliche Botschaft des Neuen Testaments. Von Jesus zur Kirche, I, Freiburg 1986.

Spiteris Y., Ecclesiologia ortodossa. Temi a confronto tra Oriente e Occidente, Bologna 2003.

Zizioulas I., Being as Communion. Studies in Personhood and the Church, London 1985.

Zizioulas I., Eucaristia e regno di Dio, Magnano 1996.

Zizioulas I., Eucharist, Bishop, Church. The Unity of the Church in the Divine Eucharist and the Bishop During the First Three Centuries, Brookline 2001.

Zizioulas I., L'essere ecclesiale, Magnano 2007.

Zizioulas I., The Early Christian Community, in: B. McGinn, J. Meyendorff, J. Leclercq (ed.), Christian Spirituality. Origins to the Twelfth Century, New York 1986, 23-43.

Zizioulas I., The Ecclesiological Presuppositions of the Holy Eucharist, „Nicolaus" 10 (1982), 333-349.

Zizioulas I., The Local Church in a Eucharistic Perspective. An Orthodox Contribution, „Mid-stream” 33 (1994), 421-434.

Ks. mgr lic. Adam Pastorczyk - sercanin, absolwent Wyższego Seminarium Misyjnego Księży Sercanów w Stadnikach i Uniwersytetu Gregoriańskiego w Rzymie; doktorant Instytutu Teologii Fundamentalnej, Ekumenii i Dialogu Uniwersytetu Papieskiego Jana Pawła II w Krakowie. 